\title{
Determinantes de compra del consumidor de prendas de vestir en la provincia de Tungurahua
}

Fecha de recepción: 2021-10-26 • Fecha de aceptación: 2021-12-08 • Fecha de publicación: 2022-02-10

Ibeth Aracelly Molina Arcos ${ }^{1}$

Tecnológico Superior Universitario España, Ecuador

ibetha.molinaa@iste.edu.ec

https://orcid.org/0000-0001-9650-1317

Jorge Oswaldo Tamayo Viera ${ }^{2}$ Tecnológico Superior Universitario España, Ecuador jorge.tamayo@iste.edu.ec https://orcid.org/0000-0002-2554-8684

Vicente Leonardo Pérez Yauli ${ }^{3}$ Estudio Jurídico Acosta \& Asociados, Ecuador p.leonardo94@yahoo.es https://orcid.org/0000-0003-1937-7820

Edison Manuel Arroba Freire ${ }^{4}$ Tecnológico Superior Universitario España, Ecuador edison.arroba@iste.edu.ec https://orcid.org/0000-0002-7196-0784

\section{RESUMEN}

La presente investigación se centra en el estudio del comportamiento del consumidor, en relación a las diferentes prendas de vestir confeccionadas. El objetivo fue identificar y categorizar los factores que determinan el consumo de prendas confeccionadas y el comportamiento de compra en la ciudad de Ambato - provincia de Tungurahua, Ecuador. 
El estudio presenta los resultados proporcionados por un modelo de investigación descriptiva con determinantes clave para la compra de prendas confeccionadas que se pueden agrupar en las categorías, (1) estilo de vida, (2) liderazgo de moda, (3) atributos económicos, y (4) contexto de consumo. La revisión ofrece una visión general sistemática del diverso conjunto de conceptos y orientaciones que se han utilizado, hasta ahora, para examinar el comportamiento de los consumidores hacia la compra de prendas confeccionadas. Los hallazgos muestran que existe un fuerte enfoque en la sostenibilidad de la ropa. El consumo sustentable ha originado el impulso de investigaciones que procuran develar tácticas que admitan la concienciación de las corresponsabilidades sociales, frente a la decisión de compra, tanto desde la perspectiva de consumidor, productor como del comerciante. El análisis se realizó a través de criterios de evaluación, aglutinando a la mayoría de los consumidores en prendas confeccionadas, productores y comerciantes.

\section{PALABRAS CLAVE: consumidor, sustentabilidad, desarrollo sostenible, comportamiento,} responsabilidad social, textil

\section{ABSTRACT}

This research focuses on the study of consumer behavior in relation to different ready-made garments. The objective was to identify and categorize the factors that determine the consumption of ready-made garments and the purchase behavior in the city of Ambato - province of Tungurahua, Ecuador.

The study presents the results provided by a descriptive research model with key determinants for ready-made garment purchase that can be grouped into the categories, (1) lifestyle, (2) fashion leadership, (3) economic attributes, and (4) consumption context. The review provides a systematic overview of the diverse set of concepts and orientations that have been used, thus far, to examine consumer behavior toward ready-made garment purchases. The findings show that there is a strong focus on the sustainability of clothing. Sustainable consumption has originated the impulse of researches that seek to unveil tactics that admit the awareness of social co-responsibilities, in front of the purchase decision, both from the perspective of the consumer, producer and retailer. The analysis was carried out through evaluation criteria, bringing together the majority of consumers in ready-made garments, producers and retailers.

KEYWORDS: consumer, sustainability, sustainable development, behavior, social responsibility, textile 


\section{Introducción}

Al hablar de la industria manufacturera, independientemente de su ubicación, ésta refiere a aquellas empresas que han dirigido todos sus recursos, sean estos humanos, financieros, materiales, tecnológicos, etc., a la transformación de materias primas en productos elaborados; dentro del proceso de producción resulta imprescindible también contar con el auxilio de personas ajenas a la industria, con el propósito de dar a conocer los productos elaboradas en otras latitudes, en esto se incluyen autoridades públicas encargadas de otorgar permisos y autorizaciones (aduanas, ministerio del ambiente), como también personas del lugar de oferta de las prendas confeccionadas.

La industria manufacturera, tanto nacional, como internacional, es concebida como un sector de significativa importancia; la comercialización de los productos ofertados por esta industria ha sido considerada como un bien de carácter significativo a nivel mundial, dado el movimiento de capital e ingresos que genera para todos los involucrados.

Otro aspecto importante de esta industria consiste en la incorporación a los diferentes procesos de producción o elaboración a la población económicamente (PEA), lo cual implica beneficios considerables de desarrollo para todos los demás sectores involucrados, como proveedores, distribuidores e intermediadores (López, 2010, citado en Ruiz et al., 2019, p. 2).

En este orden de ideas, es necesario referir también al concepto de mercado, el cual es entendido como el espacio físico en el cual generan operaciones comerciales, la muy conocida oferta demanda de productos, independientemente de su procedencia como el agro, materias primas de diferente naturaleza, productos terminados o elaborados; desde otra perspectiva, el mercado es definido como el conjunto de vendedores (intermediarios) y comparadores que desarrollan operaciones de comercio, negociando con productos de diferente naturaleza (Economía. WS, 2018, citado en Toala Checa, 2019, p. 42).

Dentro de la industria textil, producción de prendas de vestir o confeccionadas; cuando se alude a "comercio", se hacer relación al intercambio que se produce entre productores, vendedores, compradores. En esto, juega un papel preponderante los llamados atributos o características del producto como: el precio, diseño y marca; factores que inciden de forma directa en el comportamiento del cliente; por consecuencia en la toma de decisiones, dentro el proceso de adquisición e incluso exportación (Arroyave, E., \& Zapata, 2021. P. 9).

La calidad se relaciona con el conjunto de propiedades o cualidades que permiten singularizar y dar valor en términos económicos, en el caso que nos atañe, de una prenda de vestir, relacionada directamente con la confección en la que interviene el diseño, trazos, corte, costura, terminación y la percepción al tacto, respecto al tipo de material utilizado en la confección, atributos que se basan en experiencias propias o ajenas de la persona comprador o consumidor (Belmartino et al., 2016, p. 9). 
En la adquisición de prendas de vestir, como se han indicado, son muchas las características a tomarse en cuenta al momento de comprar; sin embargo, es preciso referir al diseño, precio, marca, factores que influyen decisivamente en la toma de decisión del consumidor:

a. Diseño. Permite la creación de diferentes modelos de vestuario, lo cual hace que una prenda resulte atractiva o no para el gusto del consumidor, y; por tanto, sea demandada con mayor o menor frecuencia, a esto se añade la buena confección y el acabado perfecto (Paredes Campos, 2018, p. 17).

b. Precio. Constituye un atributo de las prendas de vestir, siempre se encuentra presente al momento de realizar una adquisición, misma que se relaciona con la capacidad de compra del consumidor, quien por este medio tiene la confianza y libertad de seleccionar lo que más se apegue a su presupuesto (Patiño \& Mejía, 2019, p. 36).

c. Marca. Es una de las características que el consumidor al momento de adquirir una o varias prendas de vestir toma como referencia, la relaciona de forma estrecha con la calidad; de acuerdo al criterio de ciertos consumidores, estos prefieren adquirir productos de marca, debido a la facilidad de identificación y garantía que ofrece este tipo de prendas (Barrionuevo López, 2018, p. 33).

La industria textil a nivel nacional es una de las áreas de mayor explotación por parte de dueños y empresarios vinculados con este sector, y acogida para la población en general; este tipo de industrias se encuentran ubicadas en todo el territorio, principalmente en las provincias de Pichincha, Guayas, Azuay, Imbabura y Tungurahua (Crespo, 2015, citado en Acosta \& Tintín, 2018, p. 49).

La influencia de la industria textil es positiva, tanto en el ámbito social como económico; en razón de emplear abundante mano de obra local, nacional y actualmente dado los flujos migratorios internacional; sin embargo, ha sido también objeto de duras críticas, en razón a las condiciones desfavorables en las que las personas contratadas desarrollan sus actividades, entre estas: salarios reducidos, trabajo infantil, escasas medidas de seguridad laboral, hechos que no en todas las industrias se manifiestan, pues se cumple el adagio popular "existen excepciones", pero que a la postre perjudican por la consideración general en la sociedad (Vásquez, 2019, p. 44).

El consumidor, comprador o adquirente o como quiera que se lo conciba en el argot popular, alude a aquella $o$ aquellas personas que por diferentes circunstancias adquieren productos, bienes 0 servicios, a productores de forma directa o a distribuidores, vendedores o intermediarios de forma indirecta, con el propósito de satisfacer alguna necesidad del momento o a futuro; es decir, hace uso del producto o bien adquirido.

En la adquisición de productos, particularmente de prendas de vestir, intervienen diferentes factores que dentro de la industria comúnmente son conocidos como "atributos de las prendas confeccionadas", que comúnmente son valorados por el consumidor, y; que influyen grandemente en decisión final de compra o no de un bien o servicio. En este proceso de compra no siempre existe la intervención directa del vendedor u ofertante, como es el caso de las compras online; sin embargo, de hacerlo éste no influye decisivamente, pues, aquel no posee información real sobre 
los atributos buscados, y que motivan en el consumidor la decisión de comprar o no los productos que son ofertados en las diferentes formas y por los distintos medios (Belmartino et al., 2016, p. 8).

El comportamiento del consumidor es influenciado y determinado por diferentes variables, mismas que establecen la forma en que éste se comporta ante las distintas situaciones de consumo, que circundan alrededor y dentro de la sociedad.

En este contexto, el comportamiento del consumidor, de forma general, es entendido como el conjunto de actividades que una persona, indistintamente de su condición, realiza dentro de lo que es la selección, compra, evaluación y utilización de productos, bienes y servicios; con el propósito de satisfacer sus necesidades o deseos propios o ajenas (allegados); en estas actividades se encuentran los procesos de naturaleza mental, emocional; como también acciones físicas (Quintanilla, 2006, citado en Huete \& Zelaya, 2019, p. 27).

Complementario a lo anterior, el referido comportamiento es influenciado por una serie de factores de procedencia: social, cultural, personal y psicológica, los cuales de forma singularizada o en común influyen en la decisión final de compra, factores que según la doctrina de la materia no pueden ser controlados, pero que; son necesarios tomarlas en consideración:

a. Factores sociales. Relativos a la opinión que tienen terceras personas respecto al producto (prendas de vestir) que el consumidor pretende adquirir, su punto de vista influye de forma positiva o negativa en la toma de la decisión final. En este punto tiene papel preponderante la llamada propagando o publicidad de boca a boca, que influye decisivamente en la psiquis del consumidor (Masaquiza, 2018, p. 32).

a. Factores culturales. Relacionados estrechamente con los valores, percepciones, deseos y comportamientos; propios de la persona y sociedad, los cuales han sido transmitidos de generación en generación por la familia o instituciones (Barrionuevo López, 2018, p. 33).

a. Factores personales. Referentes a las características propias de cada individuo, estos ejercen influencia en su comportamiento como consumidor; entre aquellos se encuentran su edad, estilo de vida, profesión, situación económica, etc. (Barrionuevo López, 2018, p. 33).

a. Factores psicológicos. Concernientes a costumbres, tradiciones, apreciación de una marca reconocida, entre los principales factores de orden psicológico se encuentran la motivación, percepción, aprendizaje y creencias y actitudes (Masaquiza, 2018, p. 35).

Desde el punto de vista de la economía, el comportamiento del consumidor también es influenciado en gran o poca medida por la capacidad adquisitiva de que disponga, la que resulta determinante para que una persona se decida en la adquisición de prendas confeccionadas y a su vez considere aquellos factores ya indicados e incluso otros como la durabilidad, diseño de moda (diseñador reconocido o no), funcionalidad, facilidades de conservación, utilidad a corto, mediano y largo plazo; atributos que influyen en su razonamiento.

Las etapas que determinan el comportamiento del consumidor comprenden la pre compra, la compra y la post compra, de acuerdo con Huete \& Zelay (2019), se detallan a continuación cada una de ellas: 
a. Pre compra. Etapa en la que el consumidor identifica sus necesidades, busca información, recibe la oferta que el comercio ofrece por los diferentes medios, de acuerdo a esto toma la decisión visitar las distintas tiendas (almacenes, proveedores) que ofertan el producto demandado, realiza una evaluación de las características más sobresalientes, selecciona otras alternativas pretendiendo siempre obtener el mayor beneficio.

a. Compra. Fase en la que el consumidor ya decide en que establecimiento y con quien llevara a efecto el proceso de negociación, las condiciones que regirán en el intercambio, es influenciado fuertemente por las variables situacionales como las facilidades de crédito, de pago, garantías de con las que cuenta el producto adquirido y la factibilidad de hacerlas efectivas.

a. Post compra. Fase considerada de cierre, se produce una vez que el consumidor ha adquirido el producto, esta interrelacionada con la sensación de satisfacción o insatisfacción de la persona y consecuentemente con la cesación de la necesidad que género en primera instancia la intención y posteriormente la materialización de compra o adquisición.

En relación a las preferencias en el consumo de productos textiles, dentro de la población de consumidores existen marcada diferencias, así, las personas comprendidas entre los quince y veinte y cuatro años inclinan su atención e interés en nuevos diseños, colecciones distintas, lo que comúnmente se conoce como de temporada o de moda; no se muestran renuentes a pagar precios elevados por conseguir el producto de su preferencia; de otro lado, los consumidores comprendidos entre los cuarenta y cinco y sesenta y cuatro años, dentro de sus preferencias se encuentran las prendas en cuya confección se denote estilo, calidad y confort (PROMPERÚ, 2015, citado en Castillo Uchuya et al., 2017, p. 38).

En relación a lo anterior, en los diversos estudios desarrollados sobre el comportamiento del consumidor, las conclusiones ha permitido establecer que dentro de esta consideración existen diferencias, no todas las personas consumidoras son iguales, estas variantes tienen como origen las emociones, forma de precepción, motivaciones, necesidades e impulsos; denominas como factores internos, y; la cultura, estrato social, demografía, grupos de pertenencia; conocidos como factores externos (Ortega-Vivanco, 2020, p. 236).

Un factor preponderante y de significativa importancia, que ha influido de manera decisiva en el comportamiento del consumidor, en sus inicios constituyó la pandemia COVID 19, con efectos negativos para la población a nivel mundial, la cual afectó a todos los sectores productivos, entre ellos al manufacturero; en la mayoría de casos, trajo consigo la paralización obligatoria en la producción, en otros favoreció el emprendimiento, cambiando el giro inicial de ciertas industrias, la que con nueva visión se dedicaron a la producción de implementos de bioseguridad.

Adicional a las implicaciones negativas producto de la pandemia, se aunaron otros factores de similares características para el sector textil, particularmente para el nacional, como los altos costos de producción, obligaciones tributarias, créditos pendientes, cuentas por cobrar, competencia desleal y contrabando; hechos que, aunque a simple vista no inciden en el comportamiento del consumidor, tienen influencia en la decisión de adquirir o no un producto, bien o servicio (Sánchez et al., 2020, p. 4). 
No obstante estos hechos negativos, junto con el temor de las personas al contagio; actualmente como aspecto positivo es posible señalar que las formas de adquisición de productos, bienes y servicios ha cambiado, precios son más cómodos, los procesos son más rápidos; características que corresponden propiamente a las llamadas tiendas online; esto ha demandado todo un despliegue tecnológico, de coordinación humana, con significativos beneficios para el consumidor (Casco, 2020, citado en Rodríguez-Peña \& Gamboa-Salinas, 2021, p. 191).

\section{Metodología}

La presente investigación fue de corte cualitativo, lo cual comprendió desarrollar una actuación dinámica y sistémica en el proceso indagación y recolección de información, el estudio tomo como punto de partida una orientación teórica respecto del objeto de estudio; para esto fue necesario recurrir a estudios relacionados con el tema central de investigación, desarrollados por diferentes autores e instituciones (Escudero \& Cortez, 2018, p. 43). Fue necesario también emplear el método cuantitativo; con el propósito de adquirir conocimientos fundamentales que, permitan acercarse a la realidad del objeto de estudio de manera imparcial, mediante la recolección y análisis de datos y variables medibles (Neill \& Cortez, 2018, p. 69). Para ello se contó con el aporte de entidades como: el Observatorio Económico y Social de Tungurahua, a través de la información validada de 996 encuestas efectivas, estadísticas sector externo de la balanza comercial emitida por el Banco Central del Ecuador, el Instituto Nacional de Estadística y Censo (INEC) mediante el empleo del índice de producción de la industria manufacturera IPI-M; los cuales fueron objeto de análisis e interpretación mediante tablas estadísticas; se utilizó el método descriptivo imprescindible para caracterizar al objeto de estudio.

La investigación comprendió el periodo 2017 - 2021, dentro de la provincia de Tungurahua y demás provincias en cuyas circunscripciones existen asentamientos de empresas dedicadas a la industria manufacturera textil. 


\section{Resultados}

Figura 1.

Adquisición de productos a través de los medios electrónicos en la ciudad de Ambato

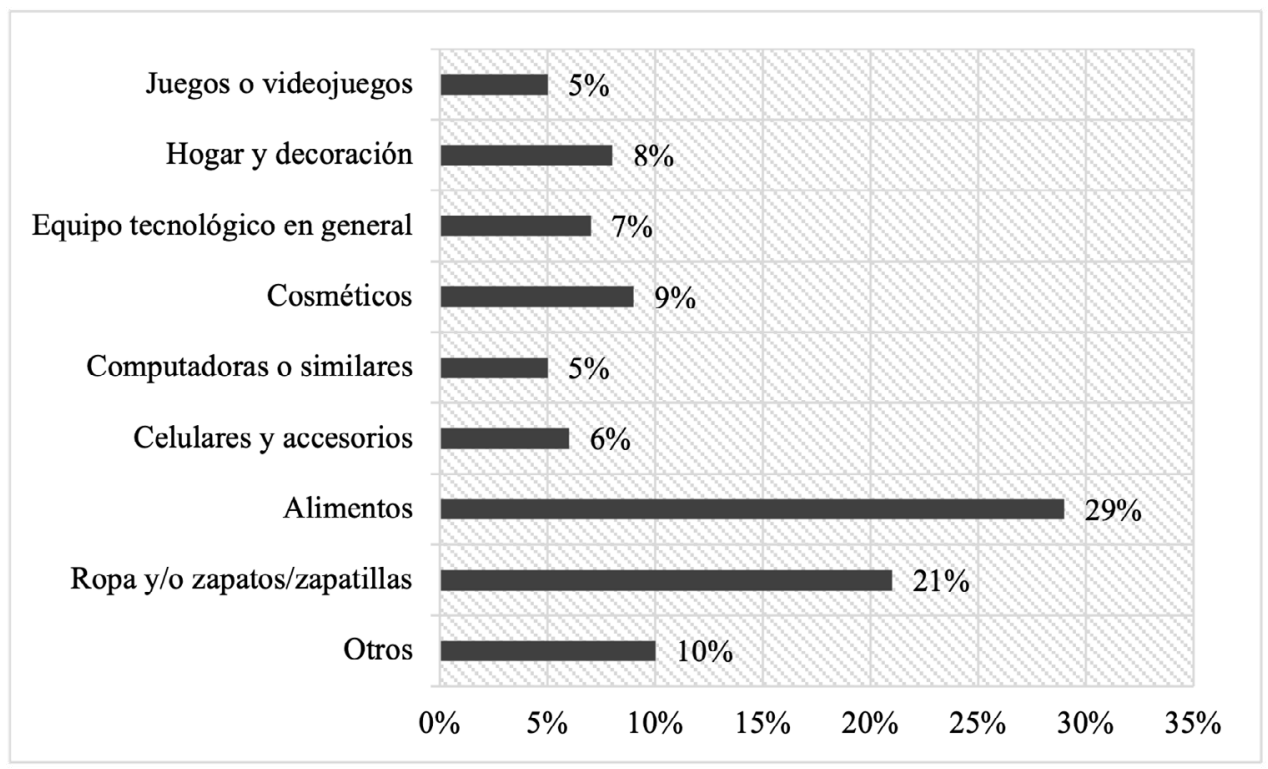

Fuente: Valle et al. (2021)

Los productos de mayor demanda, que los consumidores solicitan adquirir a través de la utilización de medios electrónicos en la ciudad de Ambato, son los alimentos (Figura 1), que se ubican en el primer lugar con el $29 \%$, seguido de la ropa, zapatos y zapatillas, que registran el $21 \%$. Los productos tecnológicos, cosméticos, celulares y accesorias también están en la lista de preferencias por parte de los clientes. 
Figura 2.

Principales maneras para adquirir un producto por parte del consumidor

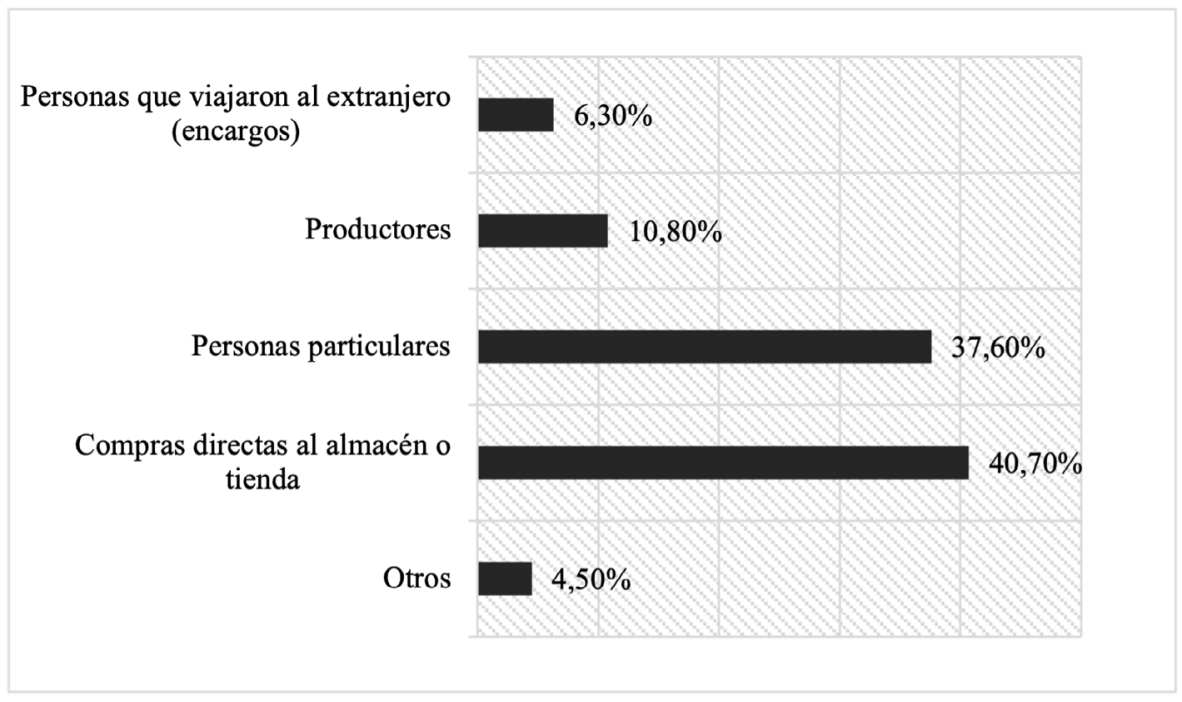

Fuente: Valle et al. (2021)

De acuerdo con la Figura 2, una de las principales características para adquirir un producto o servicio en la ciudad de Ambato, es través de la compra directa al almacén o tienda, con el $40,70 \%$. El consumidor también adquiere en gran medida los productos y servicios de personas particulares. Otras maneras que aparecen es a través de los encargos que realizan las personas que viajan al extranjero y también obteniendo el bien directamente de los productores.

\section{Figura 3.}

Medios electrónicos de compra empleados en la ciudad de Ambato

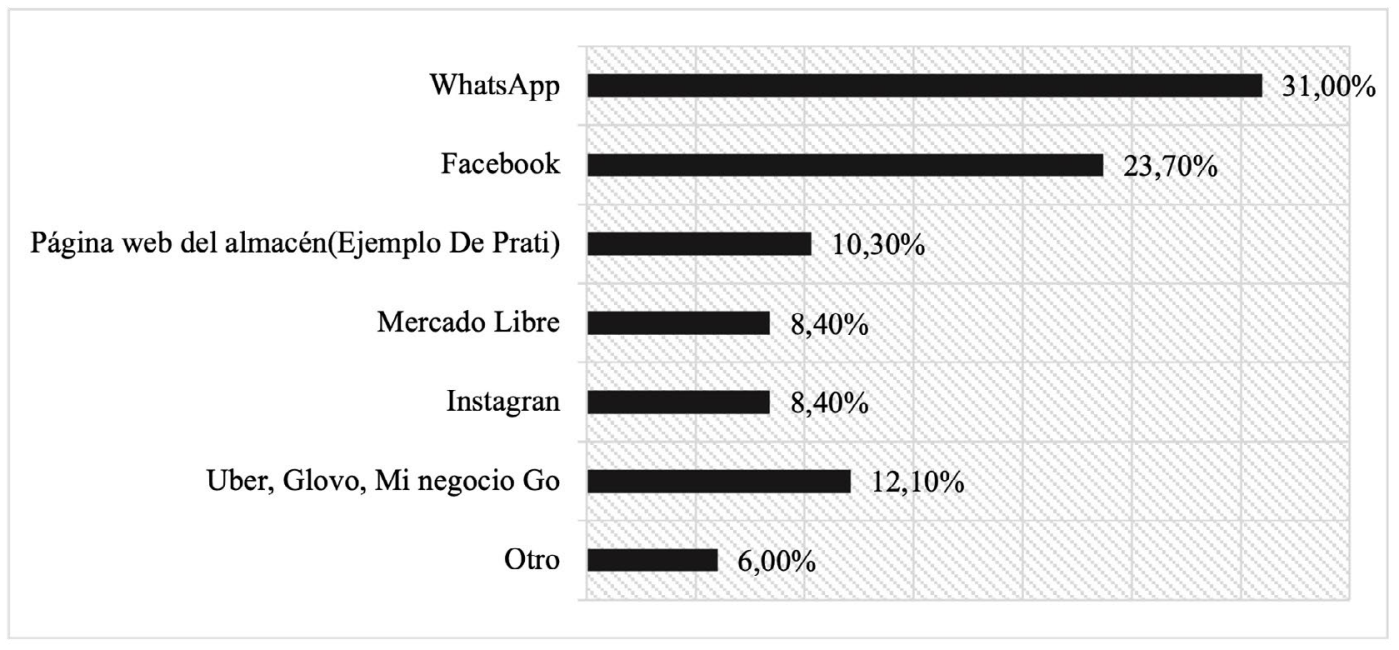

Fuente: Valle et al. (2021) 
En lo referente al comercio electrónico, el WhatsApp constituye el principal medio de compra electrónica que la gente utiliza en Ambato, con el 31,00\%, como se evidencia en la Figura 3. EI Facebook se posiciona como la segunda red social de preferencia con el $23,70 \%$. Las páginas web que mantienen diferentes almacenes dedicados a la venta de ropa y demás productos como De Prati, Etafashion, representan el 10,30\% de aceptación, de acuerdo a la información del Observatorio Económico y Social de Tungurahua (Valle et al., 2021).

Figura 4.

Concentración de empresas dedicadas a la confección de prendas de vestir

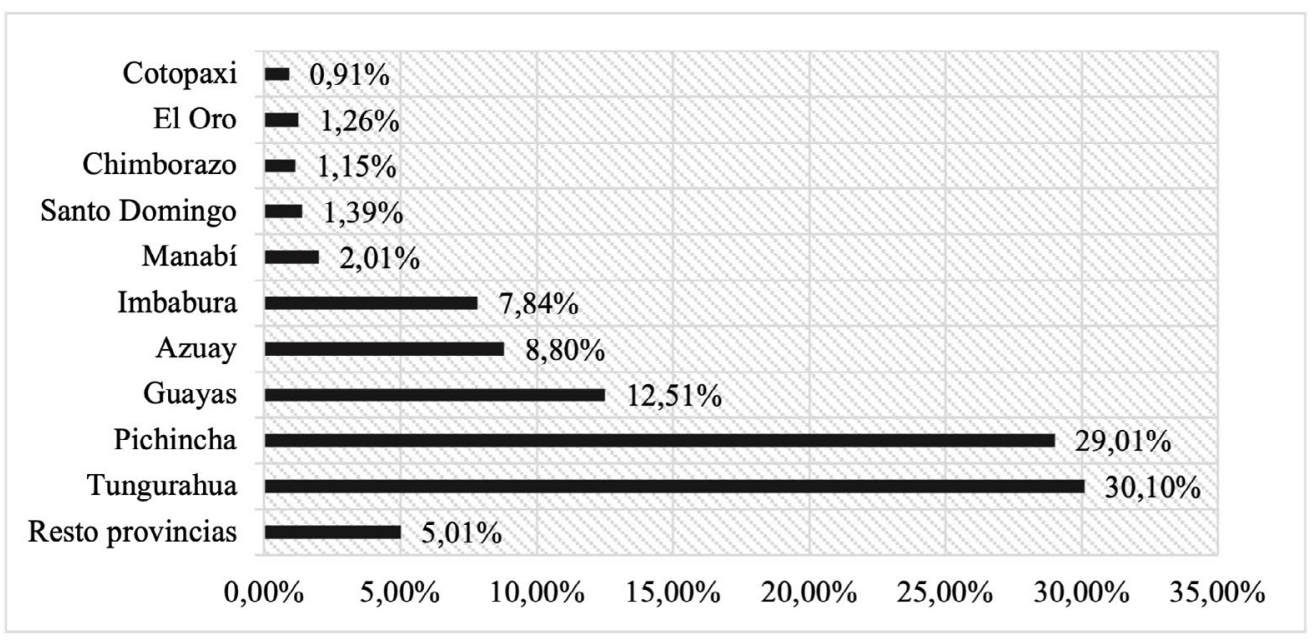

Fuente: Sánchez et al. (2020)

Tungurahua es una de las provincias que registra la mayor concentración de empresas dedicadas a la confección de prendas de vestir con el $30,10 \%$, seguido de Pichincha con el 29,01\%. Guayas se constituye en la tercera provincia con el $12,51 \%$. Cotopaxi, El Oro, Chimborazo, Santo Domingo y Manabí son las provincias que tienen poca participación en el sector de la fabricación de la ropa, como se puede observar en la Figura 4.

Tabla 1.

Exportaciones de productos textiles industrializados por partida. Miles de USD FOB

\begin{tabular}{ccccc}
\hline Partida & $\mathbf{2 0 1 7}$ & $\mathbf{2 0 1 8}$ & $\mathbf{2 0 1 9}$ & $\mathbf{2 0 2 0}$ \\
\hline 50. Seda & & & $\$ 8,70$ & \\
\hline 51. Lana y pelo fino & $\$ 451,90$ & $\$ 746,60$ & $\$ 549,70$ & $\$ 123,80$ \\
\hline 52. Algodón & $\$ 29.963,60$ & $\$ 32.566,70$ & $\$ 21.708,80$ & $\$ 3.209,50$ \\
\hline 53. Las demás fibras textiles & $\$ 25,50$ & & $\$ 9,60$ & \\
\hline 54. Filamento sintéticos & $\$ 3.675,20$ & $\$ 3.198,40$ & $\$ 3.031,80$ & $\$ 740,50$ \\
\hline 55. Fibras sintéticas & $\$ 4.154,40$ & $\$ 4.149,10$ & $\$ 4.172,10$ & $\$ 710,10$ \\
\hline 56. Guata, fieltro, tela sin & $\$ 2.007,00$ & $\$ 2.489,90$ & $\$ 3.577,30$ & $\$ 686,80$ \\
tejer & & & & \\
\hline
\end{tabular}




\begin{tabular}{ccccc}
\hline 57. Alfombras & $\$ 112,20$ & $\$ 53,10$ & $\$ 100,80$ & $\$ 15,10$ \\
\hline 58. Tejidos especiales & $\$ 183,80$ & $\$ 206,30$ & $\$ 195,60$ & $\$ 44,40$ \\
\hline 59. Telas impregnadas & $\$ 551,10$ & $\$ 332,70$ & $\$ 487,80$ & $\$ 52,20$ \\
\hline 60. Tejidos de punto & $\$ 764,20$ & $\$ 644,80$ & $\$ 658,70$ & $\$ 34,90$ \\
\hline $\begin{array}{c}\text { 61. Prendas de vestir y } \\
\text { complementos de punto }\end{array}$ & $\$ 9.920,50$ & $\$ 14.858,70$ & $\$ 15.744,30$ & $\$ 2.096,10$ \\
\hline $\begin{array}{c}\text { 62. Prendas de vestir y } \\
\text { complementos excepto de } \\
\text { punto }\end{array}$ & $\$ 4.972,00$ & $\$ 5.782,90$ & $\$ 9.415,80$ & $\$ 1.760,50$ \\
\hline 63. Los demás & $\$ 16.122,90$ & $\$ 16.294,80$ & $\$ 15.850,20$ & $\$ 4.171,00$ \\
\hline Total: & $\$ 72.904,30$ & $\$ 81.324,00$ & $\$ 75.511,20$ & $\$ 13.644,90$ \\
\hline
\end{tabular}

Fuente: Sánchez et al. (2020)

En lo referente a las exportaciones de productos industrializados en el sector textil de la confección de prendas de vestir, se contemplan 14 partidas arancelarias se muestran en la Tabla 1. El algodón representa la partida de mayor ingreso económico con un valor de 32.566,70 miles de USD FOB en el año 2018. Las prendas de vestir y complementos de punto y las prendas de vestir y complementos excepto de punto, son las partidas que registran el segundo y tercer lugar en el valor de las exportaciones.

Figura 5.

Volumen de exportaciones de prendas de vestir de fibras textiles. Miles de Tm

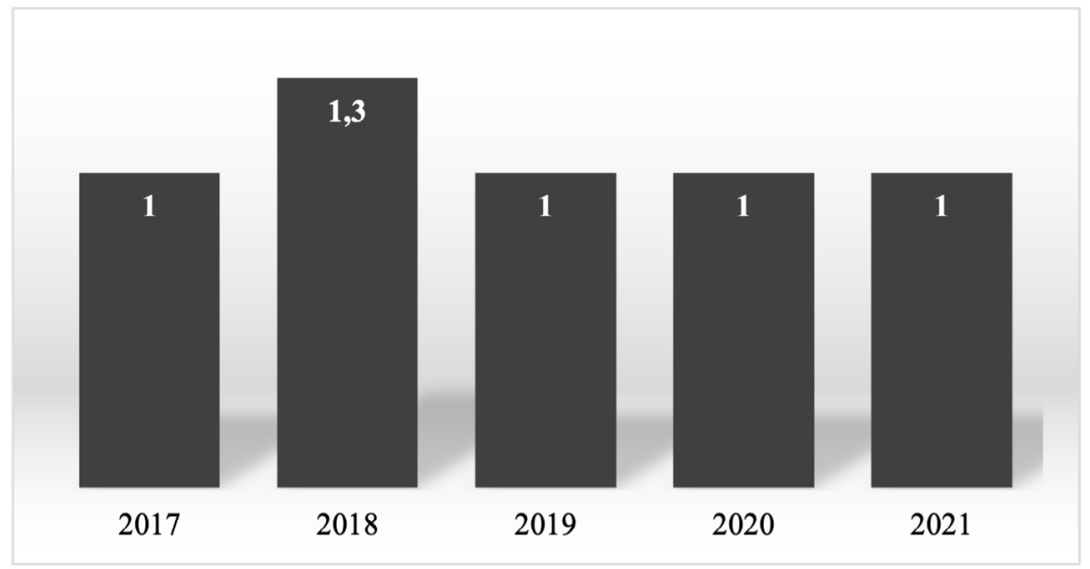

Fuente: Banco Central del Ecuador (2021)

En relación al volumen de las exportaciones de prendas de vestir de fibras textiles, el Banco Central del Ecuador registra 1,3 miles de toneladas métricas, siendo el año 2018 en donde ha existido la mayor participación en las ventas de este producto textil (Figura 5). En los años 2017, 2019, 2020 y en el primer semestre del 2021 se mantiene un promedio de 1,0 miles de toneladas métricas en las exportaciones. 
Figura 6.

Exportaciones de prendas de vestir de fibras textiles. Millones de USD

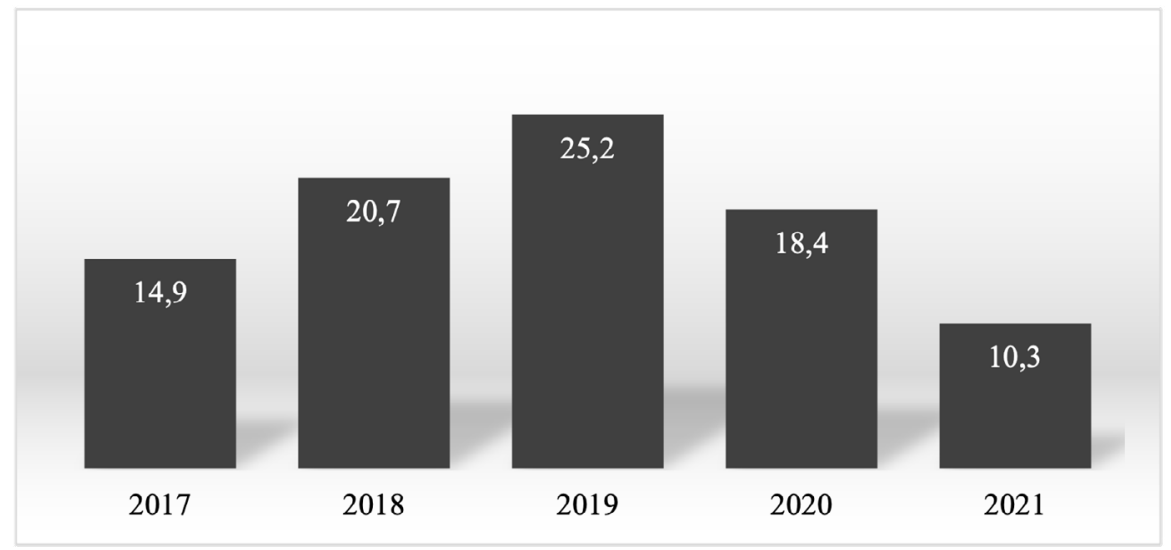

Fuente: Banco Central del Ecuador (2021)

Las exportaciones de prendas de vestir de fibras textiles efectuadas en el año 2019, alcanzaron su mayor registro con USD 25,2 millones (Figura 6). Esta exportación de producto no tradicional para el 2018 tuvo un valor de USD 20,7 millones y su tercera mejor exportación fue en el año 2020, con un volumen en ventas de USD 18,4 millones. En el primer semestre del 2021 el Banco Central del Ecuador registra exportaciones por USD 10,3 millones.

\section{Figura 7.}

Participación en valor de las exportaciones de prendas de vestir de fibras textiles

\begin{tabular}{|c|c|c|c|c|c|}
\hline \multicolumn{6}{|c|}{ Prendas de vestir de fibras textiles } \\
\hline \multicolumn{6}{|c|}{ No tradicionales $\square$} \\
\hline \multicolumn{6}{|c|}{ Tradicionales } \\
\hline \multicolumn{6}{|c|}{ No petroleras } \\
\hline \multicolumn{6}{|r|}{$100 \% 120 \%$} \\
\hline & No petroleras & Tradicionales & No trad & icionales & $\begin{array}{l}\text { Prendas de vestir } \\
\text { de fibras textiles }\end{array}$ \\
\hline -2017 & $100 \%$ & $58,30 \%$ & \multicolumn{2}{|c|}{$41,70 \%$} & $0,10 \%$ \\
\hline$\square 2018$ & $100 \%$ & $59,10 \%$ & \multicolumn{2}{|c|}{$40,90 \%$} & $0,20 \%$ \\
\hline ш2019 & $100 \%$ & $61,10 \%$ & \multicolumn{2}{|c|}{$38,90 \%$} & $0,20 \%$ \\
\hline$\square 2020$ & $100 \%$ & $58,80 \%$ & \multicolumn{2}{|c|}{$41,20 \%$} & $0,10 \%$ \\
\hline \multirow[t]{2}{*}{$\square 2021$} & $100 \%$ & $57,00 \%$ & \multicolumn{2}{|c|}{$43,00 \%$} & $0,10 \%$ \\
\hline & \multicolumn{2}{|r|}{-2018 $\quad 2019$} & $=2020$ & 2021 & \\
\hline
\end{tabular}

Fuente: Banco Central del Ecuador (2021) 
Las exportaciones no petroleras corresponden al 100\% de la participación en valor, como se puede observar en la Figura 7. Los productos tradicionales registran su mayor valor en el año 2019 con el $61,10 \%$. Los productos no tradicionales representan casi la mitad de las exportaciones totales, siendo el periodo de enero a junio del 2021 donde se contabiliza el mejor valor de participación con el $43,00 \%$. Las prendas de vestir de fibras textiles participan con el $0,10 \%$ y $0,20 \%$, respectivamente del total de las exportaciones de los productos no tradicionales.

Figura 8.

Incidencia mensual en artículos textiles y prendas de vestir

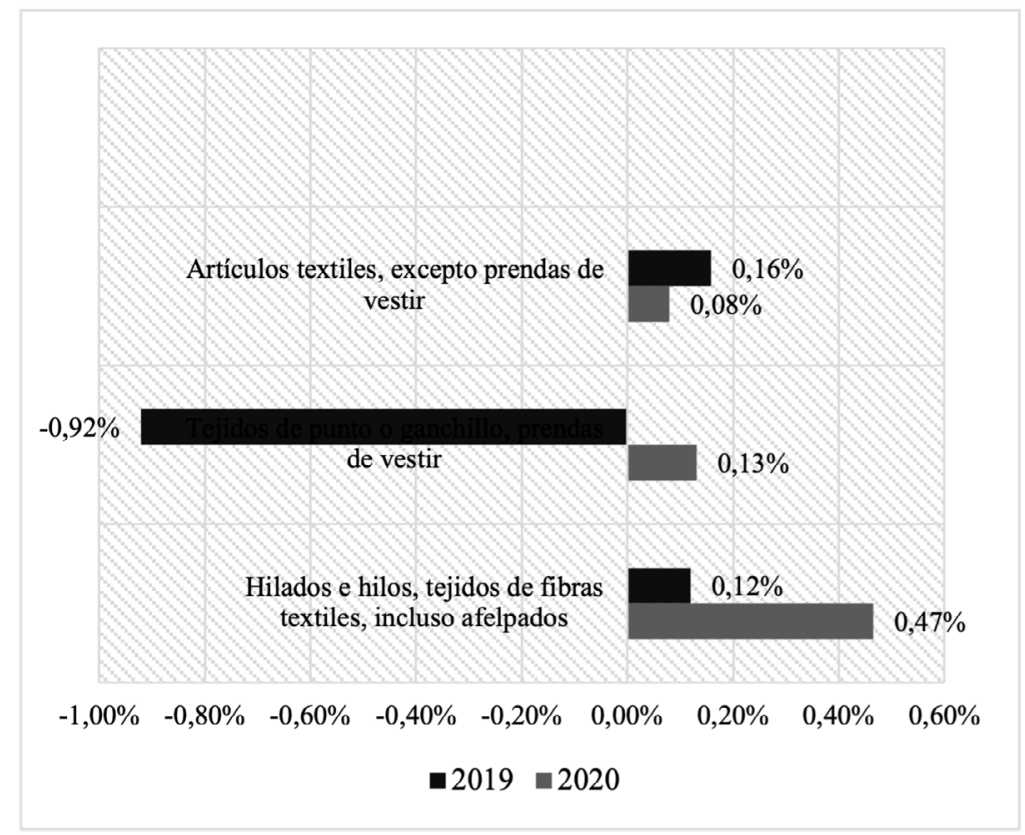

Fuente: Instituto Nacional de Estadística y Censos (2020)

En el sector de la industria manufacturera, los grupos de productos que presentaron variación positiva mensual fueron los artículos textiles excepto prendas de vestir con $0,16 \%$ en el año 2019 ; hilados e hilos, tejidos de fibras textiles incluso afelpados con 0,47\% correspondiente al 2020. De igual manera, se presentaron variación negativa mensual en los tejidos de punto o ganchillo, prendas de vestir con -0,92\% en el mes de septiembre del 2019 (Figura 8). 
Figura 9.

Exportaciones de prendas de vestir partida 61 y 62. Miles de USD FOB. Tm

\begin{tabular}{|c|c|c|c|c|}
\hline \multirow{5}{*}{$\begin{array}{l}30000,00 \\
25000,00 \\
20000,00 \\
15000,00 \\
10000,00\end{array}$} & \multicolumn{4}{|c|}{25160,10} \\
\hline & \multicolumn{3}{|c|}{20641,60} & \\
\hline & \multicolumn{3}{|l|}{14892,50} & \\
\hline & & & \\
\hline & & & 1324,00 & 3856,60 \\
\hline \multirow{2}{*}{$\begin{array}{r}5000,00 \\
0,00\end{array}$} & $c$ & - & & \\
\hline & 2017 & 2018 & 2019 & 2020 \\
\hline$\simeq$ FOB & 14892,50 & 20641,60 & 25160,10 & 3856,60 \\
\hline$\because$ TONELADAS & 945,50 & 1248,30 & 1324,00 & 191,20 \\
\hline
\end{tabular}

Fuente: Sánchez et al. (2020)

En las partidas arancelarias 61 y 62 , relacionadas a los productos textiles, las exportaciones de prendas de vestir en el año 2017 presentan variaciones de 945,50 en toneladas métricas que corresponden a 14892,50 miles de USD, como se muestra en la Figura 9. El 2019 es el año de mayor exportación de prendas de vestir con 25160 miles de USD que equivalen a 1324,00 toneladas. Hasta el mes de febrero del 2020 se han exportado un volumen de 191,20 toneladas métricas.

Tabla 2.

Exportaciones de prendas de vestir por país de destino partida 61. Miles de USD FOB

\begin{tabular}{ccccc}
\hline Países & $\mathbf{2 0 1 7}$ & $\mathbf{2 0 1 8}$ & $\mathbf{2 0 1 9}$ & $\mathbf{2 0 2 0}$ \\
\hline Alemania & 167,40 & 490,30 & 157,10 & 142,50 \\
\hline Bolivia & 820,00 & 161,40 & 960,20 & 167,20 \\
\hline Chile & 886,60 & 705,60 & 648,20 & 90,60 \\
\hline Colombia & 2202,90 & 170,80 & 1341,40 & 222,30 \\
\hline Costa Rica & 38,40 & 38,40 & 144,50 & 1,90 \\
\hline España & 114,20 & 30,60 & 58,80 & 5,00 \\
\hline Estados Unidos & 3389,10 & 7526,90 & 6511,20 & 799,30 \\
\hline Guatemala & 129,80 & 93,80 & 317,40 & 7,50 \\
\hline México & 786,50 & 364,80 & 256,80 & 7,20 \\
\hline Países Bajos & 1,80 & 187,70 & 282,20 & 97,50 \\
(Holanda) & & & & \\
\hline Panamá & 181,50 & 470,30 & 299,90 & 47,70 \\
\hline Perú & 456,90 & 1446,80 & 2888,90 & 290,50 \\
\hline Puerto Rico & 126,10 & 114,70 & 277,50 & 3,50 \\
\hline Suiza & 80,00 & 156,00 & 276,70 & 65,00 \\
\hline Venezuela & 8,50 & 0,00 & 0,00 & 0,00 \\
\hline
\end{tabular}

Fuente: Sánchez et al. (2020) 
Estados Unidos fue el país de mayor destino en las exportaciones de las prendas de vestir, alcanzando sus mayores ventas en el año 2018, con 7526,90 miles de USD (Tabla 2). El total de exportaciones en el año 2017 en las prendas de vestir y complementos de punto que corresponden a la partida 61 , fue de $\$ 9.389,10$ miles de USD que representan un volumen de 466,4 toneladas métricas. Hasta el mes de febrero del 2020 se registran 1.947,70 miles de USD y un total de 85,9 Tm, siendo los principales países de destino en el año 2020 los Estados Unidos con 72,4\%, Panamá 5,6\%, Perú 3,4\% y Guatemala 2,3\% del total de las exportaciones.

Tabla 3.

Exportaciones de prendas de vestir por país de destino partida 62. Miles de USD FOB

\begin{tabular}{|c|c|c|c|c|}
\hline Países & 2017 & 2018 & 2019 & 2020 \\
\hline Bolivia & 135,70 & 147,40 & 169,30 & 30,30 \\
\hline Colombia & 129,70 & 68,90 & 140,60 & 3,10 \\
\hline Costa Rica & 66,60 & 46,50 & 216,00 & 3,30 \\
\hline España & 757,60 & 755,70 & 624,20 & 16,80 \\
\hline Estados Unidos & 1577,10 & 1926,00 & 5399,50 & 1274,70 \\
\hline Guatemala & 274,90 & 660,50 & 245,00 & 41,20 \\
\hline Italia & 91,40 & 102,50 & 119,00 & 0,00 \\
\hline $\begin{array}{l}\text { Países Bajos } \\
\text { (Holanda) }\end{array}$ & 310,80 & 224,40 & 256,80 & 7,40 \\
\hline Panamá & 104,40 & 160,60 & 127,90 & 99,10 \\
\hline Perú & 458,40 & 535,80 & 984,70 & 60,20 \\
\hline Alemania & 27,30 & 18,80 & 73,60 & 2,10 \\
\hline Canadá & 234,80 & 215,70 & 190,40 & 12,10 \\
\hline Chile & 342,20 & 499,70 & 401,40 & 17,20 \\
\hline México & 33,50 & 27,30 & 93,20 & 0,00 \\
\hline
\end{tabular}

Fuente: Sánchez et al. (2020)

El total de exportaciones de prendas de vestir en el año 2019 fue de $\$ 9.415,8$ miles de USD y 466,4 Tm. Hasta el mes de febrero del año 2020 se registran $1.760,5$ miles de USD y 85,9 Tm, siendo los principales países de destino en 2020 los Estados Unidos con 72,4\%, Panamá 5,6\%, Perú 3,4\% y Guatemala 2,3\%. Las ventas de prendas de vestir y complementos excepto punto que corresponden a la partida 62 tuvo su principal facturación con los Estados Unidos en el año 2019 con 5399,50 miles de USD, tal como se puede observar en la Tabla 3.

\section{Conclusiones}

En la actualidad, el comportamiento del consumidor de la industria textil se ha transformado y la manera de consumo a nivel mundial también. El sector manufacturero, específicamente las partidas 61 y 62 relacionadas a las prendas de vestir y complementos de punto, prendas de vestir 
y complementos, excepto punto, registraron cifras positivas en las exportaciones de los productos no tradicionales.

Las prendas de vestir de fibras textiles son las más representativas en los mercados internacionales con una participación en valor del $0,10 \%$ y $0,20 \%$, del total de las exportaciones de los productos textiles nacionales, siendo los Estados Unidos el país de destino con mayor demanda.

Como parte de las conclusiones se determina que la promoción y venta de ropa se canaliza a través del comercio electrónico, siendo el WhatsApp la principal aplicación como medio de compra electrónica que la gente utiliza en Ambato, y es precisamente la provincia de Tungurahua quien registra la mayor concentración de empresas dedicadas a la confección de prendas de vestir en el mercado.

En el presente artículo se reveló el punto trascendental y con ello el compromiso que ha despertado, o por mencionarlo de una forma más reveladora, la conciencia con respecto al medio ambiente y la responsabilidad social. De tal manera que se pueda entender que la sostenibilidad y sustentabilidad ya no es un tema aislado a la empresa, forma parte fundamental de la misma, a la cual los consumidores en prendas confeccionadas, productores y comerciantes deben sumarse. 


\section{Referencias}

Acosta Rivera, C., \& Tintín Caiza, A. (2018). Factores de innovación para el sector textil, provincia de Tungurahua. [Tesis de grado, Universidad Técnica de Ambato]. http://repositorio.uta.edu.ec/jspui/handle/123456789/27768

Arroyave, E., \& Zapata, V. (2021). Percepciones, emociones y atributos más relevantes al explorar un catálogo de ropa casual femenina por parte de las mujeres entre 26 y 50 años, de estratos 3 y 4 en la ciudad de Medellín. [Tesis de maestría, Universidad Eafit]. http://hdl.handle.net/10784/30145

Banco Central del Ecuador. (2021). Estadísticas sector externo. Evolución de la balanza comercial. https://contenido.bce.fin.ec/documentos/Estadisticas/SectorExterno/BalanzaPagos/balanzaComercial/indicepp.htm

Barrionuevo López, M. (2018). El branding corporativo como identidad en el estudio del comportamiento del consumidor en el sector textil del Cantón Ambato. [Tesis de grado, Universidad Técnica de Ambato]. Repositorio UTA http://repositorio.uta.edu.ec/jspui/handle/123456789/28437

Belmartino, A., Liseras, N., \& Berges, M. (2016). ¿Qué atributos busca el consumidor en prendas de vestir? Un análisis aplicado al mercado de la indumentaria en Argentina. Tec Empresarial, 10(1), 7-18. https://www. scielo.sa.cr/pdf/tec/v10n1/1659-3359-tec-10-01-7.pdf

Castillo Uchuya, B., Trujillo Herrera, W., \& Zapata Valle, G. (2017). El comportamiento del consumidor británico frente a productos textiles provenientes de Perú y China. [Tesis de grado, Universidad Peruana de Ciencias Aplicadas] http://hdl.handle.net/10757/621425

Escudero Sánchez, C., \& Cortez, L. (2018). Técnicas y métodos cualitativos para la investigación científica. Editorial UTMACH. http://repositorio.utmachala.edu.ec/handle/48000/12501

Huete, M., \& Zelaya, C. (2019). El comportamiento del consumidor en el crecimiento de mercado de la Mipyme textil Toti, en la ciudad de Estelí, durante el año 2018 y primer trimestre del año 2019. [Tesis doctoral, Universidad Nacional Autónoma de Nicaragua].

Instituto Nacional de Estadística y Censos. (2020). Directorio de Empresas y establecimientos.http://www.ecuadorencifras.gob.ec/directoriodeempresas/

Masaquiza, M. (2018). Factores que determinan la demanda en el sector textil en la comunidad de Llicacama en la parroquia de Salasaka. [Tesis de grado, Universidad Técnica de Ambato. http://repositorio.uta.edu. ec/jspui/handle/123456789/27281

Neill, D., \& Cortez, L. (2018). Procesos y Fundamentos de la Investigación Científica. Editorial UTMACH. http:// repositorio.utmachala.edu.ec/handle/48000/12498 
Ortega-Vivanco, M. (2020). Efectos del Covid-19 en el comportamiento del consumidor: Caso Ecuador. RETOS. Revista de Ciencias de la Administración y Economía, 10(20), 233-247. https://doi.org/10.17163/ret. $\underline{\mathrm{n} 20.2020 .03}$

Paredes Campos, S. (2018). Diseño, patronaje y proceso de confección de pantalones para damas para diferentes ocasiones. [Tesis de grado, Universidad Nacional de Educación Enrique Guzmán y Valle]. http:// repositorio.une.edu.pe/handle/UNE/3239

Patiño, M., \& Mejía, I. (2019). Valores de los consumidores de las marcas studio F y Ela. Universidad Ices. https://repository.icesi.edu.co/biblioteca digital/bitstream/10906/85437/1/TG02655.pdf

Rodríguez-Peña, V., \& Gamboa-Salinas, J. (2021). COVID 19 factor de cambio en la conducta del consumidor. Digital Publisher, 6(3) 188-200. https://doi.org/10.33386/593dp.2021.3.561

Espinosa Ruiz, C. G., Muyulema Allaica, J. C., Sánchez Macías, R. A., \& Usca Veloz, R. B. (2019). Los retos sostenibilistas de las Pymes textileras de la provincia de Tungurahua - Ecuador. Revista Internacional de Investigación e Innovación Tecnológica, 7 (38), 1-20. http://riiit.com.mx/apps/site/files/los retos sostenibilistas jcarlos..pdf

Sánchez, A., Vayas, T., Mayorga, F., \& Freire, C. (2020). Manufactura sector textil prendas de vestir. Observatorio Económico y Social de Tungurahua. Universidad Técnica de Ambato https://blogs.cedia.org.ec/obest/ wp-content/uploads/sites/7/2020/06/Diagn\%C3\%B3stico-sector-textil-prendas-de-vestir-corregido-mayo-2020.pdf

Toala Checa, B. (2019). Análisis de la percepción del mercado en función de la industria textil de las marcas ecuatorianas. [Tesis de grado, Universidad de Guayaquil]. http://repositorio.ug.edu.ec/handle/redug/47098

Valle, T., Sánchez, A., Vayas, T., Mayorga F., \& Freire, C. (2021). Comercio electrónico en Ambato. Observatorio Económico y Social de Tungurahua. https://blogs.cedia.org.ec/obest/wp-content/uploads/sites/7/2021/05/ Comercio-electronico-en-Ambato.pdf 
Copyright (c) 2022 Ibeth Aracelly Molina Arcos, Jorge Oswaldo Tamayo Viera, Vicente Leonardo Pérez Yauli y Edison Manuel Arroba Freire

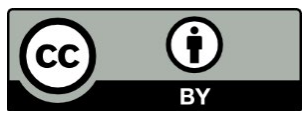

Este texto está protegido bajo una licencia internacional Creative Commons 4.0.

Usted es libre para Compartir-copiar y redistribuir el material en cualquier medio o formato - y Adaptar el documento - remezclar, transformar y crear a partir del material-para cualquier propósito, incluso para fines comerciales, siempre que cumpla las condiciones de Atribución. Usted debe dar crédito a la obra original de manera adecuada, proporcionar un enlace a la licencia, e indicar si se han realizado cambios. Puede hacerlo en cualquier forma razonable, pero no de forma tal que sugiera que tiene el apoyo del licenciante o lo recibe por el uso que hace de la obra.

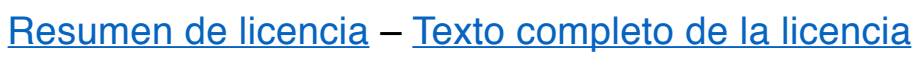

